\title{
Microscopic model, spin wave theory, and competing orders in double perovskites
}

\author{
G. Jackeli* \\ Institut Laue Langevin, B. P. 156, F-38042, Grenoble, France
}

\begin{abstract}
We present a microscopic theory of carrier-induced ferrimagnetism in metallic double perovskite compounds such as $\mathrm{Sr}_{2} \mathrm{FeMoO}_{6}$ and $\mathrm{Sr}_{2} \mathrm{FeReO}_{6}$ which have recently attracted intense interest for their possible applications to magnetotransport devices. The theory is based on an effective "Kondolike" Hamiltonian treated here within the large- $S$ expansion. We find that depending on the value of the carrier density the ground state is either a ferrimagnet or a layered antiferromagnet. The ferrimagnetic state has a robust half-metallic electronic structure. The transition to antiferromagnetic phase is first order accompanied with the regime of phase separation. We study spin wave spectrum including quantum corrections and find strongly enhanced quantum effects in the vicinity of zero-temperature phase transition.
\end{abstract}

PACS numbers:75.10.Lp, 75.30.Ds, 75.30.Vn

The recent discovery of the room temperature magnetoresistance in double perovskite compounds ${ }^{1}$ has generated intense interest in these materials because of their potential importance as magnetotransport devices as well as their rich and challenging properties. For applications metallic ferrimagnetic (FiM) compounds such as $\mathrm{Sr}_{2} \mathrm{FeMoO}_{6}$ and $\mathrm{Sr}_{2} \mathrm{FeReO}_{6}$ are of particular interest, because both have high magnetic transition temperature $\left(T_{\mathrm{c}} \simeq 420\right.$, and $\simeq 400 \mathrm{~K}$, respectively) and their electronic structures have been suggested to be halfmetallic. ${ }^{1}$ Despite an enormous interest in half-metallic ferromagnets, most of the theoretical studies until now have been only by $a b$ initio methods. ${ }^{2-5}$

In this letter we formulate a microscopic theory of the carrier-induced ferrimagnetism in this family of magnetic compounds. The theory is based on a minimal model Hamiltonian, in which only the lowest energy charge fluctuations, coupled to the local moments, are retained, and those which are moved to high energies due to strong correlations, are disallowed. The model has two parameters and allows for reliable analytical treatment of the physically relevant situation.

In the double perovskite structure $\mathrm{Fe}$ and Mo/Re ions form two face centered cubic sublattices and each pair of nearest-neighbor lattice sites is occupied by two different ions. In the ionic picture, $\mathrm{Fe}$ is in the $3+$ valence state and half-filled $d$-shell forms a $S=5 / 2$ local moment. The Mo is in $5+$ state with one $t_{2 g}$ electron in $4 d$-shell. ${ }^{2}$ This extra electron, which hybridizes with the same orbital states at the neighboring iron sites, is responsible for the system's lowest energy charge excitations. ${ }^{6}$ In the classical picture, only a spin down electron, with respect to a neighboring Fe local moment, can hop to this Fe site. For a spin up electron this hopping is blocked by the Pauli principle, as at the the iron site the spin up states are already all occupied. Thus, it is the exclusion principle, which couples the itinerant and the local spins kinematically. This contrasts with the case of double-exchange systems, in which the intra-atomic Hund's rule is responsible for the coupling between the two subsystems. ${ }^{7}$ In the magnetic double perovskites, as in double exchange magnets, ordering of local moments occurs due to the reduction of the kinetic energy. The kinetic energy in the former case is minimized when the local moments are parallel to each other and antiparallel to itinerant spins, and this causes the ferrimagnetism.

Model Hamiltonian. - We now turn to a minimal model Hamiltonian reflecting the above discussion. We retain only low energy charge excitations in the system, which correspond to the processes $\left(\mathrm{Fe} d^{5}, \mathrm{~B}^{\prime} d^{n}\right) \leftrightarrow$ $\left(\mathrm{Fe} d^{6}, \mathrm{~B}^{\prime} d^{n-1}\right)\left(\mathrm{B}^{\prime}\right.$ stands for $\left.\mathrm{Mo} / \mathrm{Re}\right)$. The Fe ions are treated as localized spins $S=5 / 2$ and extra electrons from nonmagnetic Mo/Re ions as charge carriers. There are $n=1$ and $n=2$ carriers per unit cell in Mo and Re compounds, respectively. When a carrier is placed at a site with core spin $S=5 / 2$, the total spin $\mathcal{S}$ can take two possible values $\mathcal{S}=2$ and $\mathcal{S}=3$. However, the maximal allowed spin for six electrons in a $d$-shell is $\mathcal{S}=2$. To project out the $\mathcal{S}=3$ spin state we introduce infinite local antiferromagnetic (AFM) coupling $J \rightarrow \infty$ between core and itinerant spins. ${ }^{8}$ The carriers occupy three degenerate $t_{2 g}\left(d_{x y}, d_{y z}, d_{y z}\right)$ orbital states. In the cubic lattice the $t_{2 g}$-transfer matrix is diagonal in the orbital space and is non-zero only in the corresponding plane. A minimal Hamiltonian can be written as a sum of three two dimensional (2D) terms $\mathcal{H}=H_{x y}+H_{x z}+H_{y z}$, where each term corresponds to a given orbital state. All three terms have the same form given by (we further omit orbital index for simplicity)

$$
\begin{aligned}
H & =-t \sum_{\langle i j\rangle \sigma}\left[d_{i \sigma}^{\dagger} \bar{d}_{j \sigma}+\text { H.c. }\right]+\sum_{i} \Delta_{\mathrm{CT}} n_{i}-\sum_{j} \Delta_{\mathrm{CT}} \bar{n}_{j} \\
& +\sum_{i} J\left[\mathbf{S}_{i} \mathbf{s}_{i}-A n_{i}\right]
\end{aligned}
$$

where the first term describes an electron hopping between nearest-neighbor $\mathrm{Fe}$ and $\mathrm{Mo} / \mathrm{Re}$ ions, labeled by $i$ and $j$, respectively. The operators $d_{i \sigma}\left(n_{i}\right)$ and $\bar{d}_{j \sigma}$ $\left(\bar{n}_{j}\right)$ corrsepond to $\mathrm{Fe}$ and $\mathrm{Mo} / \mathrm{Re}$ sublattices, respectively, $2 \Delta_{\mathrm{CT}}=E\left(\mathrm{Fe} d^{6}, B^{\prime} d^{0}\right)-E\left(\mathrm{Fe} d^{5}, B^{\prime} d^{1}\right)$ is a charge transfer gap, and $\mathbf{S}_{i}\left(\mathbf{s}_{i}\right)$ stands for the localized (itinerant) spin. The last term with $A=(S+1) / 2$ is chosen so 
that the exchange energy vanishes for a state with $\mathcal{S}=2$.

We now consider the ferromagnetic (FM) arrangement of local moments, which corresponds to the ground state of Hamiltonian (1) for carrier density $n<n_{1}$ (see below). In the limit $J \rightarrow \infty$, we restrict the Hilbert space to spin eigenstates with maximum allowed spin $\mathcal{S}$ at a given site. Thus, the magnetic ion is in a state either with $\mathcal{S}=S$, without an extra electron, or of $\mathcal{S}=S-1 / 2$, with an extra electron. The site states are labeled by $|\mathcal{S}, M\rangle$ and $M$ is $z$-projection of the spin $\mathcal{S}$. Considering low lying excitations we can retain only the states with $M=\mathcal{S}$ and $M=\mathcal{S}-1$. Moerover, as the value of the core spin is relatively large $S=5 / 2$ we perform the $1 / S$ expansion around the ordered state. Then the local Hilbert space consists of four states: $|S, S\rangle$, $|S, S-1\rangle,\left|S-\frac{1}{2}, S-\frac{1}{2}\right\rangle$, and $\left|S-\frac{1}{2}, S-\frac{3}{2}\right\rangle$. To describe transitions between these states we introduce magnon and fermion operators as $B^{\dagger}|\mathcal{S}, M\rangle=|\mathcal{S}, M-1\rangle$ and $D_{\downarrow}^{\dagger}|S, M\rangle=\left|S-\frac{1}{2}, M-\frac{1}{2}\right\rangle$, respectively. Through corresponding matrix elements, one can find the following relations $d_{\downarrow}=D_{\downarrow}\left[1-B^{\dagger} B / 4 S-B^{\dagger} B /\left(32 S^{2}\right)\right]$, and $d_{\uparrow}=-D_{\downarrow} B^{\dagger} / \sqrt{2 S}$ to order $1 / S^{2}$. The state generated by $D_{\uparrow}$ corresponds to $\mathcal{S}=S+1 / 2$ and is projected out.

As a next step, we express the Hamiltonian (1) in terms of the new operators, diagonalize its band part, and arrive in the momentum space to the following expression $H=H_{0}+H_{1}+H_{2}$, where

$$
\begin{aligned}
H_{0} & =\sum_{\mathbf{k}}\left\{E_{\mathbf{k}}\left[a_{\mathbf{k} \downarrow}^{\dagger} a_{\mathbf{k} \downarrow}-b_{\mathbf{k} \downarrow}^{\dagger} b_{\mathbf{k} \downarrow}\right]-\Delta_{\mathrm{CT}} \bar{d}_{\mathbf{k} \uparrow}^{\dagger} \bar{d}_{\mathbf{k} \uparrow}\right\} \\
H_{1} & =\frac{1}{\sqrt{2 S N}} \sum_{\mathbf{k}, \mathbf{q}}\left\{\bar{d}_{\mathbf{k}-\mathbf{q} \uparrow}^{\dagger}\left[N_{\mathbf{k}, \mathbf{q}} a_{\mathbf{k} \downarrow}+M_{\mathbf{k}, \mathbf{q}} b_{\mathbf{k} \downarrow}\right] B_{\mathbf{q}}^{\dagger}+\text { H.c. }\right\} \\
H_{2} & =\frac{1}{4 S N}\left[1+\frac{1}{8 S}\right] \sum_{\mathbf{k}, \mathbf{p}, \mathbf{q}} B_{\mathbf{q}}^{\dagger} B_{\mathbf{q}+\mathbf{k}-\mathbf{p}}\left\{P_{\mathbf{k}, \mathbf{p}}^{a a} a_{\mathbf{k} \downarrow}^{\dagger} a_{\mathbf{p} \downarrow}\right. \\
& \left.+P_{\mathbf{k}, \mathbf{p}}^{b b} b_{\mathbf{k} \downarrow}^{\dagger} b_{\mathbf{p} \downarrow}+P_{\mathbf{k}, \mathbf{p}}^{a b} a_{\mathbf{k} \downarrow}^{\dagger} b_{\mathbf{p} \downarrow}+P_{\mathbf{p}, \mathbf{k}}^{a b} b_{\mathbf{k} \downarrow}^{\dagger} a_{\mathbf{p} \downarrow}\right\}
\end{aligned}
$$

where $a_{\mathbf{k} \downarrow}=u_{\mathbf{k}} D_{\mathbf{k} \downarrow}-v_{\mathbf{k}} e^{i k_{x}} \bar{d}_{\mathbf{k} \downarrow}, \quad b_{\mathbf{k} \downarrow}=v_{\mathbf{k}} D_{\mathbf{k} \downarrow}+$ $u_{\mathbf{k}} e^{i k_{x}} \bar{d}_{\mathbf{k} \downarrow}$, and $u(v)_{\mathbf{k}}=\sqrt{\left[1 \pm \Delta_{\mathrm{CT}} / E_{\mathbf{k}}\right] / 2}$. The first term $H_{0}$, corresponding to the classical limit $(S \rightarrow \infty)$, describes the band structure of the system. The electronic structure of each orbital state is composed of the three bands: bonding $\left(b_{\mathbf{k} \downarrow}\right)$ and antibonding $\left(a_{\mathbf{k} \downarrow}\right)$ of down spin electrons, and nonbonding $\left(\bar{d}_{\mathbf{k} \uparrow}\right)$ of Mo/Re up spin electrons. $E_{\mathbf{k}}=\sqrt{\varepsilon_{\mathbf{k}}^{2}+\Delta_{\mathrm{CT}}^{2}}$ and $\varepsilon_{\mathbf{k}}=2 t\left(\cos k_{x}+\right.$ $\cos k_{y}$ ) for the $d_{x y}$-orbital. For $n$ carriers per unit cell each bonding band of a given orbital is $n / 3$-filled and fully polarized. Given this band structure, the system is half-metallic for arbitrary model parameters $\left(t, \Delta_{\mathrm{CT}}\right)$ and for any realistic value of carrier density. The last two terms $H_{1}$ and $H_{2}$ [see Eq.(2)] are due to the quantum nature of the core spins. Spin up electrons, localized in the classical picture, can hop thanks to the quantum nature of the local moments, but leaving a trace of spin deviations along their trajectories. This process is described by $H_{1}$. The last term $H_{2}$ describes fluctuations of the local moment generated by the hopping of spin down electrons. The corresponding vertices are given by $N_{\mathbf{k}, \mathbf{q}}=\varepsilon_{\mathbf{k}-\mathbf{q}} u_{\mathbf{k}}, M_{\mathbf{k}, \mathbf{q}}=\varepsilon_{\mathbf{k}-\mathbf{q}} v_{\mathbf{k}}$, $P_{\mathbf{k}, \mathbf{p}}^{a a}=-\varepsilon_{\mathbf{k}} v_{\mathbf{k}} u_{\mathbf{p}}-\varepsilon_{\mathbf{p}} u_{\mathbf{k}} v_{\mathbf{p}}, P_{\mathbf{k}, \mathbf{p}}^{b b}=\varepsilon_{\mathbf{k}} u_{\mathbf{k}} v_{\mathbf{p}}+\varepsilon_{\mathbf{p}} v_{\mathbf{k}} u_{\mathbf{p}}$, and $P_{\mathbf{k}, \mathbf{p}}^{a b}=\varepsilon_{\mathbf{p}} u_{\mathbf{p}} u_{\mathbf{k}}-\varepsilon_{\mathbf{k}} v_{\mathbf{k}} v_{\mathbf{p}}$.

Spin wave theory. - We now turn to analysis of the spin wave spectrum. The magnon Green function is obtained from the Dyson equation $D_{\mathbf{q}, \omega}=\left[\omega-\Sigma_{\mathbf{q}, \omega}\right]^{-1}$. Where magnon self-energy $\Sigma=\sum_{i} \Sigma_{i}$ and $i=x y, x z, y z$ refers to a given orbital channel. We note that to the order we consider the magnon self-energy does not mixes the different orbital channels. This is due to the fact that the electron propagator as well as the magnon-electron vertecies are diagonal in the orbital space. To order $1 / S$, the self-energy $\Sigma$ includes the second order perturbative contribution from $H_{1}$ as well as first order contribution from $\mathrm{H}_{2}$ (see first two diagrams in Fig 1.) (The analytical form of $\Sigma$ is lengthy and will be presented elsewhere.) Here we discuss the results. There are two branches of spin excitations, like those of localized ferrimagnets, the gapless Goldstone mode and the optical mode. ${ }^{9}$ In addition to this, there exists a Stoner continuum of spin-flip particle-hole excitations.

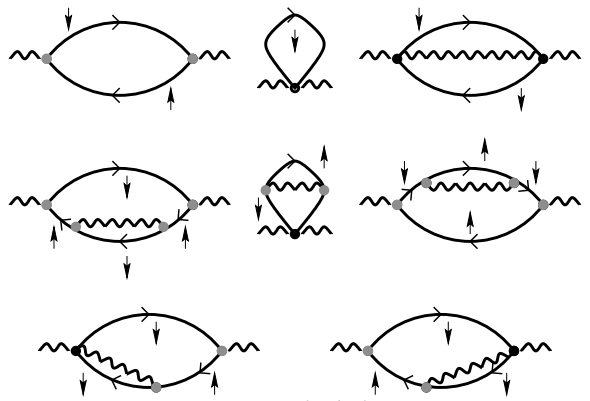

FIG. 1. The leading order $(1 / S)$ and next-to-leading order $\left(1 / S^{2}\right)$ spin wave self-energies. Wave (solid) line stands for the magnon (electron) propagator. The small arrow denotes an electron spin and gray (black) dot stands for the magnon-electron vertex proportional to $1 / \sqrt{S}(1 / S)$.

We below focus on the low energy mode. The spectrum of the magnons at the quasi-classical level is Heisenberglike $\omega_{\mathbf{q}}=16 S J_{1}\left[1-\gamma_{1 \mathbf{q}}\right]+16 S J_{2}\left[1-\gamma_{2 \mathbf{q}}\right]$, where $J_{1}$ and $J_{2}$ are nearest and next-nearest neighbor exchange couplings, respectively, $\gamma_{1 \mathbf{q}}=\left(\cos q_{x} \cos q_{y}+\right.$ $\left.\cos q_{x} \cos q_{z}+\cos q_{y} \cos q_{z}\right) / 3$, and $\gamma_{2 \mathbf{q}}=\left(\cos ^{2} q_{x}+\right.$ $\left.\cos ^{2} q_{y}+\cos ^{2} q_{z}\right) / 3$. The carrier-induced exchange energies are given by $J_{1(2)}=t^{2} /\left(16 S^{2} N\right) \sum_{\mathbf{k}} \bar{\gamma}_{1(2)} n_{\mathbf{k}} / E_{\mathbf{k}}$, where $\bar{\gamma}_{1}=2 \cos k_{x} \cos k_{y}, \bar{\gamma}_{2}=\cos 2 k_{x}+\cos 2 k_{y}$ and $n_{\mathbf{k}}$ is a Fermi distribution function. We also note that there is no Landau damping of spin waves at this order.

In Fig. 2(a) we show carrier density dependence of the spin stiffness $\mathcal{D}=16 S\left(J_{1}+J_{2}\right)$ for different values of $\Delta_{\mathrm{CT}}$. At a fixed density the stiffness scales with the bandwidth: $\mathcal{D} \sim t$ for $t \gg \Delta_{\mathrm{CT}}$ and $\mathcal{D} \sim t^{2} / \Delta_{\mathrm{CT}}$ for $t \ll \Delta_{\mathrm{CT}}$. As a function of density the spin stiffness exhibits strongly nonmonotonic behavior. It shows a maximum at optimal filling $n_{\text {opt }} \simeq 1$ and then drops to zero 
at some critical density $n_{c r}{ }^{10}$

We now analyze quantum corrections to the harmonic spectrum discussed above. These corrections are generated by the magnon self-energy diagrams proportional to $1 / S^{2}{ }^{11}$ They are governed by fermionic excitations and are thus different from those in insulating magnets. At this order magnon self-energy includes fourth and second order contributions from $H_{1}$ and $H_{2}$, respectively, as well as mixed type terms. Graphically they are presented in Fig. 1. From the structure of the diagrams one easily verifies that renormalization of the electron propagator due to the scattering of spin waves as well as all relevant vertex corrections are consistently taken into account at this order. After collecting all the diagrams in this order one recovers the Goldstone theorem, that illustrates the consistency of the approximation.

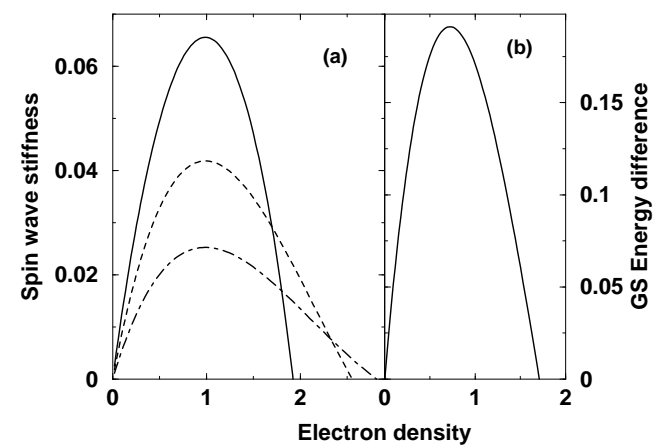

FIG. 2. (a) Spin wave stiffness (in units of $t=1$ ) versus carrier density, for different values of $\Delta_{\mathrm{CT}}\left(\Delta_{\mathrm{CT}}=0,4\right.$, and 8, solid, dashed and dotted-dashed lines, respectively); (b) Ground state (GS) energy difference (in units of $t$, and for $\Delta_{\mathrm{CT}}=0$ ) between AFM-II and ferrimagnetic states versus carrier density.

The quantum corrections generate two main effects in the magnon spectrum: (i) they give rise to spin wave damping in the ground state, and (ii) they modify the semi-classical dispersion law of magnons. The first nonzero contribution to magnon damping $\Gamma_{\mathbf{q}}$ is proportional to $1 / S^{3}$ and is due to the spin wave scattering of carrier density fluctuations. To evaluate damping, one has to replace the bare magnon propagator in the selfenergy diagrams by that obtained in harmonic approximation (see Golosov in Ref. 11). In the long wave-length limit one finds $\Gamma_{\mathbf{q}} \propto\left\langle f_{k_{\mathrm{F}}}\right\rangle t q^{6} / S^{3}$, where $\left\langle f_{k_{\mathrm{F}}}\right\rangle$ denotes the Fermi surface average of $f_{\mathbf{k}}=\left(\sin k_{x}+\sin k_{y}\right)^{2}$.

Figure 3 shows magnon dispersion obtained semiclassically (solid line) and with quantum corrections included (dashed line), for carrier densities $n=1$, Fig.3(a), and $n=1.8$, Fig.3(b). In the case of optimal carrier density, $n=1$, the quantum effects are small and practically do not alter harmonic dispersion law. However, for a higher carrier density the quantum effects become strongly pronounced and result in a dramatic modification of the harmonic spectrum. They cause stiffening of the long-wave length excitations and strong softening of the zone boundary magnons [see Fig. 3(b)].

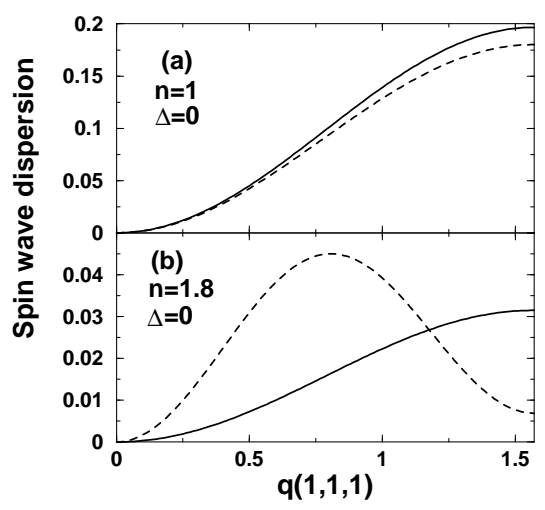

FIG. 3. Spin wave spectrum in [111] direction (in units of $t=1$ ) versus momentum. Solid and dashed lines represent the harmonic and renormalized magnon dispersion law, respectively.

Ground state phase diagram. - As we have already mentioned, there is a critical carrier density $n_{c r}$ at which the spin wave stiffness vanishes [see Fig. 2(a)]. This indicates that there exist another type of magnetic ordering which competes with FiM order and becomes favorable as $n \rightarrow n_{c r}$. The most direct route to identify the symmetry of new magnetic order is to find the wave vector at which spin wave spectrum becomes unstable. The linear theory can not answer this question, because the harmonic dispersion vanishes identically in [111] direction, and no special soft mode is singled out at this order. The problem can be resolved by considering quantum effects. The tendency of the zone boundary magnons to soften with increasing $n$ [see Fig. 3(a) and 3(b)] can be interpreted as a precursor effect of a transition to a layered magnetic ordering with wave-vector $\mathbf{Q}=\pi / 2[1,1,1]$. The latter consists of alternating $\{111\}$ FM planes. The magnetic moments of neighboring planes can be either misaligned by an angle $\theta$, which is the case of canted magnetic structure, or aligned antiparallel to form type-II antiferromagnetic (AFM-II) ordering. Hence, there are two possible scenarios for the phase transition: (a) the transition is second order and is to a canted state or (b) transition is first order to AFM-II ordering. To decide which of these two is realized we have evaluated and compared the kinetic energy of carriers in FM, canted and AFM-II background of core spins. We have found that canted state never minimizes the energy and hence is never stabilized.

In Fig. 4 (left panel) we show 2D (001) cut of AFM-II magnetic structure. In this magnetic structure carriers are confined within the 1D FM stripes and have the dispersion law $\varepsilon_{k}=-2 \sqrt{2} t \cos k$ (the factor $\sqrt{2}$ is due to the two possible path of the charge transfer between nearestneighbor magnetic ions). Figure 2(b) shows the difference between the energies $(\Delta E)$ of the AFM-II and FiM states versus carrier density for $\Delta_{\mathrm{CT}}=0$ (the same qualitative behavior is realized for finite values of $\Delta_{\mathrm{CT}}$ ). As it is seen in this figure, with increasing carrier density $\Delta E$ shows the same trend, not coincidently, as the spin wave 
stiffness [see Fig. 2(a)], and vanishes at carrier concentration $\tilde{n}_{c r}$ slightly smaller than $n_{c r}$ suggested from linear spin wave theory. For $n>\tilde{n}_{c r}$ carriers gain more kinetic energy in AFM-II state. This consideration suggests the first order transition from ferrimagnetic to AFM-II state. To investigate the possibility of phase separation accompanying the first order transition we have evaluated the ground state thermodynamic potential $\Omega=E-\mu n$ for homogenous ferrimagnetic and AFM-II phases as a function of chemical potential $\mu$. The homogenous state is destabilized with respect to phase separation when $\Omega$ of the ferrimagnetic phase becomes larger than that of AFM-II state. The value of $\mu$ at which this takes place gives the lower $n_{1}$ and upper $n_{2}$ boundaries of carrier density for the phase separation regime. We find that the carrier density $n_{1}$, at which which the phase separation occurs, is smaller then the critical carrier densities suggested form the above analysis, the following inequality holds $n_{1}<\tilde{n}_{c r}\left(n_{c r}\right)<n_{2}$. For $\Delta_{\mathrm{CT}}=0$ we find $n_{1} \simeq 1.5$ and $n_{2} \simeq 2$.

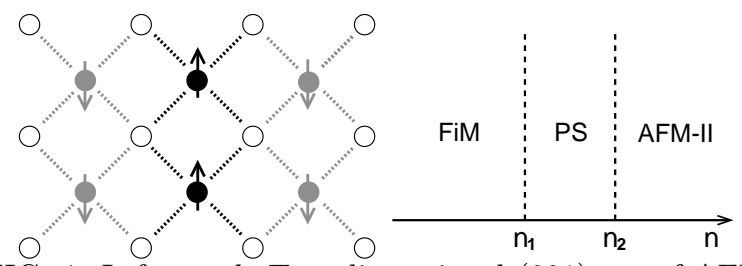

FIG. 4. Left panel: Two dimensional (001) cut of AFM-II structure. Filled (opened) circles denote $\mathrm{Fe}(\mathrm{Mo} / \mathrm{Re})$ ions and big arrows stand for the core spins. Black (grey) dashed lines indicate the allowed path for spin down (up) electrons; Right panel: ground state phase diagram of model (1).

Based on the above analysis, in Fig. 4 (right panel) we present ground state phase diagram of Hamiltonian (1). For carrier density $n<n_{1}$ the ground state is ferrimagnet, for $n_{1}<n<n_{2}$ the systems phase separates (PS) in ferrimagnetic and AFM-II phases. The volume fraction occupied by FiM and AFM-II phases are given by $v_{1}=\left(n-n_{1}\right) /\left(n_{2}-n_{1}\right)$ and $v_{2}=\left(n_{2}-n\right) /\left(n_{2}-n_{1}\right)$, respectively. Here, we point out, that in the real systems, long-range Coulomb interaction, not considered here, most likely will prevent the phase separation at macroscopic scale resulting in the nanoscale domains. ${ }^{12}$ We have also analyzed the stability of homogenous AFMII phase within the linear spin wave theory and found that this magnetic ordering is indeed stable for $n \gtrsim n_{2}$.

So far, we have not considered the effect of direct AFM exchange $J^{\prime}$ between the local moments. At the semi-classical level, an effect of $J^{\prime}$ is to reduce carrierinduced FM exchange. However, in the compounds with large transfer gap $\Delta_{\mathrm{CT}}$, FM exchange can be largely suppressed and AFM exchange, if strong enough, can stabilize AFM-II magnetic ordering. Considering the metallic compounds with fully polarized carriers we have also neglected on-site Coulomb repulsion $U$ between charge carriers on $3 d$ level of Fe-ions. (As at Mo/Re ions electrons reside in more extended $4 d / 5 d$ level this interaction is much smaller and can be neglected. ${ }^{2,4}$ ) This approximation is fully justified for $\mathrm{Sr}_{2} \mathrm{FeMO}_{6}$ compound that has one charge carrier per Fe-Mo cell and hence the Fe-ion is effectively quarter filled. Therefore the probability of generating doubly-occupied states is low, and the effect of the Gutzwiller projector on the uncorrelated wave function is expected to be negligible. As for the $\mathrm{Sr}_{2} \mathrm{FeReO}_{6}$ which has two charge carrier per formula unit, the on-site $U$, if it is not largely screened in the metallic state, may suppress the charge fluctuations and renormalize electronic caracteristics of the system, such as an effective carrier density and their band-width. However, as far as magnetic properties of the systems are concerned, we expect that Coulomb repulsion can only change our quantitative but not qualitative predictions. The spin wave spectrum as well as its carrier density dependence can be directly verified by inelastic neutron scattering experiments on Mo and Re based compounds.

We thank N. Andrei, E. I. Kats, D. I. Khomskii, D.E. Logan, and $\mathrm{Ph}$. Nozières for their interests and useful discussions. Earlier discussion with D. D. Sarma is gratefully acknowledged. We are indebted to M. E. Zhitomirsky for continuous valuable discussions and to $\mathrm{T}$. Ziman for a critical reading of the manuscript.

* On leave from E. Andronikashvili Institute of Physics, Georgian Academy of Sciences, Tbilisi, Georgia.

${ }^{1}$ K.-I. Kobayashi et al., Nature (London) 395, 677 (1998); K.-I. Kobayashi et al., Phys. Rev. B 59, 11159 (1999).

${ }^{2}$ D. D. Sarma et al., Phys. Rev. Lett. 85, 2549 (2000); S. Ray et al., ibid. 87, 097204 (2001).

${ }^{3}$ Z. Fang, K. Terakura, and J. Kanamori, Phys. Rev. B 63, 180407 (2001).

${ }^{4}$ I. V. Solovyev, Phys. Rev. B 65, 144446 (2002).

${ }^{5}$ T. Saitoh et al., Phys. Rev. B 66, 035112 (2002).

6 This is supported by the observation that there are no evidences of presence of $\mathrm{Fe}^{4+}\left(d^{4}\right)$ in the ground state.

${ }^{7}$ Since completing this work we have become aware of work, D. D. Sarma, Cur. Opin. Solid State Mat. Sci. 5, 261 (2001), in which a similar point was made.

${ }^{8}$ The limit $J \rightarrow \infty$ has been also discussed by E. L. Nagaev (cond-mat/0204308), however in a different context. We thank D. I. Khomskii for pointing this to us.

${ }^{9}$ H. Kaplan, Phys. Rev. 86, 121 (1952).

10 This is in agreement with the dynamical mean-filed study of a somewhat extended Hamiltonian by A. Chattopadhyay and A. J. Millis, Phys. Rev. B 64, 024424 (2001).

11 To the same order, the analysis for double exchange magnets has been done in D. I. Golosov, Phys. Rev. Lett. 84, 3974 (2000); N. Shannon and A. V. Chubukov, Phys. Rev. B 65, 104418 (2002)).

12 E. Dagotto, Nanoscale Phase Separation and Colossal Magnetoresistance (Springer-Verlag, Berlin, 2003). 